17;01

\title{
Перколяционный переход в углеродном композите на основе фуллеренов и терморасширенного графита
}

\author{
(C) В.И. Берёзкин ${ }^{1}$, В.В. Попов ${ }^{2}$ \\ ${ }^{1}$ Научно-исследовательский центр экологической безопасности РАН, \\ Санкт-Петербург, Россия \\ ${ }^{2}$ Физико-технический институт им. А.Ф. Иофффе РАН, \\ Санкт-Петербург, Россия \\ E-mail: v.berezkin@inbox.ru
}

(Поступила в Редакцию 10 мая 2017 г.)

\begin{abstract}
Исследована электропроводность углеродного композита на основе фуллеренов $\mathrm{C}_{60}$ и терморасширенного графита в диапазоне относительных содержаний компонентов 0-100\%. Образцы получены путем термообработки в вакууме в диффузионно-адсорбционном процессе исходных дисперсных смесей и их дальнейшего холодного прессования. С увеличением доли фуллеренов удельное сопротивление образцов постепенно растет, а по достижении определенных концентраций $\mathrm{C}_{60}$ наблюдается резкий переход из проводящего состояния в изолирующее. Интерпретация результатов в рамках теории перколяции позволяет оценить порог протекания (как относительное содержание графита) величиной 4.45 wt.\%, критический индекс электропроводности величиной 1.85 (что характерно для трехмерных двухкомпонентных неупорядоченных сред, в том числе имеющих поры).
\end{abstract}

DOI: 10.21883/FTT.2018.01.45309.148

\section{1. Введение}

Способность углерода образовывать множество разнообразных структур обеспечивает возможность создавать и широко использовать основанные на нем материалы, которые зачастую обладают уникальными электрическими, механическими, адсорбционными и другими свойствами $[1,2]$. Активные исследования объектов, базирующихся на таких углеродных разновидностях, как фуллерены, нанотрубки, графены, различные формы так называемого аморфного углерода, пополняют знания об особенностях состояний углерода с разным типом и уровнем упорядочения атомов и способствуют поиску реального практического применения указанных углеродных модификаций.

Расширить диапазон полезных качеств различных углеродных объектов позволяет разработка композитов, свойства которых определяются свойствами всех компонентов (наряду с углеродом это обычно полимеры, керамика, металлы). Такие композиционные материалы уже давно применяются на практике [1]. Если говорить о перспективах, то создание, например, композитов типа углерод-полимер с улучшенными механическими характеристиками и повышенной электропроводностью нередко связывают с углеродными нанотрубками [3].

Увеличить электропроводность самих углеродных материалов позволяет их модификация, что может приводить, в частности, графит, алмаз, фуллерены, нанотрубки, органические соединения даже к сверхпроводимости [1]. В фуллеренах, как хорошо известно, она возникает при их интеркалировании атомами ряда металлов [4]. Не менее хорошо известно также и то, что такие сверхпроводящие фуллереновые конденсаты (кри- сталлы, поликристаллические порошки, пленки) крайне нестабильны. В воздушной среде они быстро (за доли секунды [5]) теряют сверхпроводимость вследствие окисления металлических примесей.

Возможность создания стабильных сверхпроводников на основе фуллеренов была продемонстрирована в работах [6,7], где были представлены композиты типа углерод-углерод, в которых фуллерены и легирующие примеси размещаются в углеродной матрице, причем химически связываются с ней. При использовании примеси натрия получена сверхпроводимость при температурах $T \leq 15 \mathrm{~K}$, которая не исчезает при хранении материала на воздухе при комнатной температуре. Указанные композиты синтезированы с помощью „алмазной“ технологии, иными словами - путем воздействия высоких давлений и температур на исходные механические смеси, которые включали фуллерены, углеводородные связующие вещества (нафталин $\mathrm{C}_{10} \mathrm{H}_{8}$ и др.), а также соединения, содержащие легирующие элементы. Правда, для подобной технологии, как хорошо известно, характерна слабая воспроизводимость результатов в силу существенных неоднородностей целого ряда параметров в зоне синтеза.

В работах $[8,9]$ представлена аналогичная (но нелегированная) система, где в качестве связующего вещества использован терморасширенный графит (ТРГ). Образцы при весовых соотношениях $\mathrm{C}_{60}$ :ТРГ от $1: 16$ до 16:1 получены термообработкой исходных смесей в диффузионно-адсорбционном процессе с дальнейшим холодным прессованием и отжигом. Оказалось, что между фуллеренами и окружением образуются ковалентные связи, материал достаточно однороден и характеризуется довольно высокой электропроводностью. 
В настоящей работе электропроводность системы $\mathrm{C}_{60}-$ ТРГ исследована в более широком диапазоне соотношений указанных компонентов, что позволило наблюдать переход композита в высокоомное состояние.

\section{2. Экспериментальные образцы и измерения}

Для синтеза композита исходные дисперсные смеси ТРГ и поликристаллических порошков $\mathrm{C}_{60}$ в откачанных и отпаянных стеклянных ампулах обрабатывали в течение нескольких часов при $550-650^{\circ} \mathrm{C}$ (что значительно выше температуры сублимации $\mathrm{C}_{60}$, примерно равной $400^{\circ} \mathrm{C}$ ). Затем полученный продукт при комнатной температуре $\left(T_{R}\right)$ прессовали при давлении $0.7 \mathrm{GPa}$ в пластины диаметром $13 \mathrm{~mm}$ и толщиной $\sim 1 \mathrm{~mm}$. Более подробно технология описана в работе [8].

Получены материалы при 12 разных исходных весовых соотношениях $\mathrm{C}_{60}$ и ТРГ: $1: 16,1: 8,1: 4, \ldots$, $128: 1$ (т. е. при содержании $\mathrm{C}_{60}$ от 5.9 до $99.2 \mathrm{wt} . \%$ ). Кроме того, раздельным прессованием дисперсных ТРГ и $\mathrm{C}_{60}$ при том же давлении $0.7 \mathrm{GPa}$ получены монолитные образцы из чистых ТРГ и $\mathrm{C}_{60}$. В итоге имелся набор пластин 14 составов, из которых вырезали образцы нужной формы. Образцы отжигу не подвергали, поскольку он, как показано в работах $[8,9]$, практически не влияет на механизмы проводимости в образцах с ТРГ, а лишь создает дополнительные электронные ловушечные центры, что приводит к некоторому росту удельного электросопротивления $\rho$ (на $\sim 50 \%)$. В образцах без ТРГ (т.е. в чисто фуллереновых монолитах) отжиг не проводили во избежание их возможного разрушения вследствие деполимеризации $\mathrm{C}_{60}$.

Удельное электросопротивление $\rho$ исследовали с помощью стандартной четырехзондовой методики. Измерены либо зависимости $\rho(T)$ в широком диапазоне температур от $T=4.2 \mathrm{~K}$ до $T_{R}$, либо величины $\rho$ в одной точке $T_{R}$.

\section{3. Полученные результаты}

На рис. 1 показана зависимость удельного сопротивления материала при $T_{R}$ от относительного содержания $\mathrm{C}_{60}$. Видно, что имеются образцы двух типов низкой и высокой величиной $\rho$, причем переход от одних к другим осуществляется скачком.

Относительно низкоомными оказались образцы при соотношениях $\mathrm{C}_{60}:$ ТРГ $\leq 16$. Здесь наименьшим удельным сопротивлением обладает чистый прессованный ТРГ с $\rho=1.65 \mathrm{~m} \Omega \cdot \mathrm{cm}$. При внедрении в ТРГ фуллеренов и увеличении их доли величина $\rho$ монотонно возрастает и достигает $\rho=0.25 \Omega \cdot \mathrm{cm}$ при соотношении $\mathrm{C}_{60}:$ ТРГ $=16: 1$. Иными словами, рост содержания фуллеренов от 0 до $94.1 \mathrm{wt} \%$ приводит к постепенному росту сопротивления на два порядка.

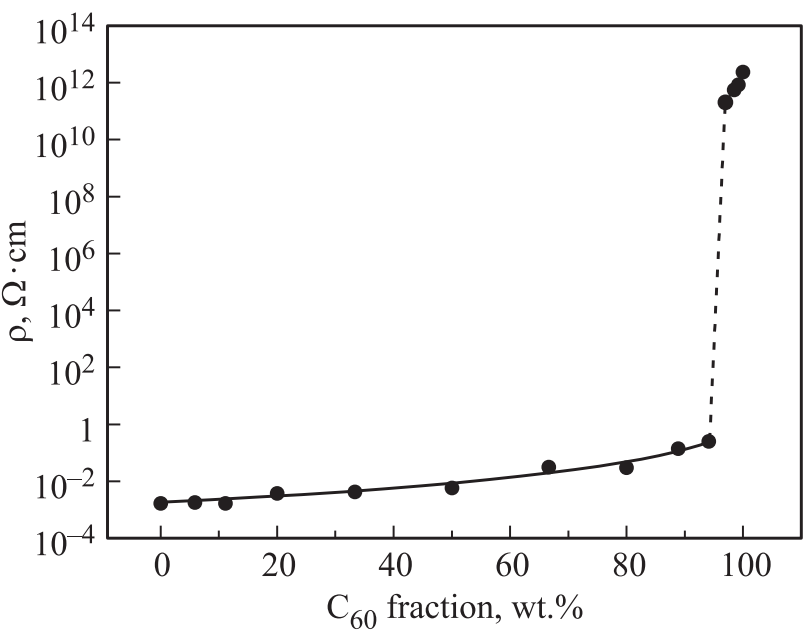

Рис. 1. Удельное электросопротивление материала при $T_{R}$ в зависимости от относительного содержания $\mathrm{C}_{60}$.

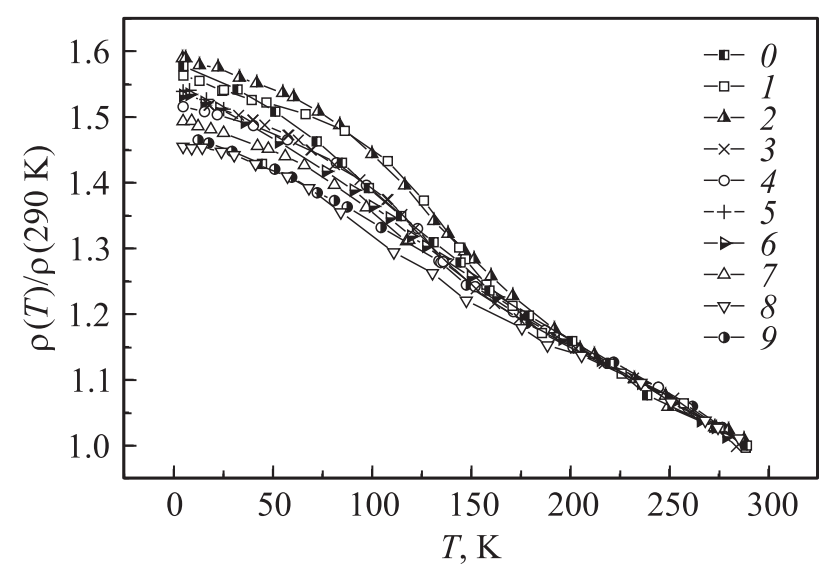

Рис. 2. Относительные температурные зависимости $\rho(T) / \rho(290 \mathrm{~K})$ образцов с разным соотношением компонентов; 0 - образец без $\mathrm{C}_{60}$, т.е. чистый прессованный ТРГ; $1-9-$ образцы со следующими соотношениями $\mathrm{C}_{60}$ :ТРГ по весу (в скобках указано относительное содержание $\mathrm{C}_{60}$ в wt.\%): $1-1: 16$ (5.9), $2-1: 8$ (11.1), $3-1: 4$ (20.0), $4-1: 2$ (33.3), $5-1: 1$ (50.0), $6-2: 1$ (66.7), 7 - 4:1 (80.0), $8-8: 1$ (88.9), $9-16: 1$ (94.1). Для удобства восприятия часть экспериментальных точек удалена.

При дальнейшем росте относительной доли $\mathrm{C}_{60}$ сопротивление скачком увеличивается на 12 порядков, достигая $\rho=2.03 \cdot 10^{11} \Omega \cdot \mathrm{cm}$ при соотношении $\mathrm{C}_{60}:$ ТРГ $=32: 1$. Иными словами, при увеличении содержания $\mathrm{C}_{60}$ всего лишь от 94.1 до 97.0 wt.\% наблюдается резкий переход из проводящего в изолирующее состояние. При дальнейшем также относительно небольшом росте концентрации $\mathrm{C}_{60}$ (на 3 wt.\%, от 97 до $100 \mathrm{wt} . \%$ ) $\rho$ продолжает расти, увеличиваясь еще на порядок и достигая максимального значения $\rho=2.38 \cdot 10^{12} \Omega \cdot \mathrm{cm}$ в чистом прессованном $\mathrm{C}_{60}$. Так что высоким электросопротивлением характеризовались образцы при соотношениях $\mathrm{C}_{60}$ :ТРГ $>16$, а также чистый монолитный $\mathrm{C}_{60}$. 
Измерения зависимостей $\rho(T)$ в низкоомных образцах показали, что $\rho$ растет при снижении температуры. При этом на начальном участке от $T_{R}$ и ниже удельное сопротивление растет довольно медленно, затем по мере дальнейшего снижения температуры скорость несколько увеличивается, а при самых низких значениях $T$ рост $\rho$ опять замедляется. Такая форма оказалась практически одинаковой для всех зависимостей $\rho(T)$, наблюдаемых в относительно низкоомных образцах, рис. 2. Здесь эти зависимости показаны нормированными на величину $\rho$ при $T_{R}$ (в данном случае $290 \mathrm{~K}$ ). Видно, что все кривые подобны друг другу, при этом разброс значений $\rho(T) / \rho(290 \mathrm{~K})$ при низких температурах в разных по составу образцах не превышает $\pm 5 \%$ от относительно среднего значения.

\section{4. Обсуждение результатов}

Тот факт, что зависимости $\rho(T)$ на рис. 2 по характеру одинаковы для всех образцов (без фуллеренов, при невысоком, среднем и довольно большом их содержании) дает основания полагать, что в низкоомных образцах вид кривых $\rho(T)$ определяется главным образом углеродной матрицей, в которой размещается $\mathrm{C}_{60}$, a также технологией и ее параметрами. Измерения коэффициента Холла показали [9], что концентрация носителей тока (в основном дырок) в этих образцах $\sim 10^{18}-10^{20} \mathrm{~cm}^{-3}$, т.е. приближается к величинам, наблюдаемым в металлах.

Показанные на рис. 2 кривые $\rho(T)$ во многом подобны таким же зависимостям, наблюдающимся в некоторых других неупорядоченных углеродных системах, например, в аналогичном фуллереновом композите $[6,7]$ или биоморфном углероде [10]. В этих объектах кривые $\rho(T)$ удовлетворительно описываются теорией квантовых поправок к проводимости, применяемой для разупорядоченных металлических систем [11]. В наших низкоомных образцах зависимости $\rho(T)$ имеют более сложный характер, однако их также можно интерпретировать в рамках указанной теории. При этом оказалось [9], что в области от $T=4.2 \mathrm{~K}$ до $T \approx 90-180 \mathrm{~K}$ (в разных образцах) преобладают эффекты слабой локализации, а при более высоких температурах в большей мере проявляются электрон-электронные взаимодействия. Величины подвижности достигают значений $\mu_{h} \approx 30 \mathrm{~cm}^{2}(\mathrm{~V} \cdot \mathrm{s})^{-1}$, что на порядок больше, чем в биоморфном углероде, и на два порядка больше, чем в аналогичном фуллереновом композите. Это, по-видимому, связано с более совершенной структурой углеродной матрицы, сформированной из ТРГ, что и позволяет ей определять проводимость низкоомных образцов. Что касается высоких значений $\rho$ в исследуемом материале, то они наблюдаются в образцах с самыми малыми концентрациями ТРГ и в чисто фуллереновых монолитах, в которых углеродная матрица отсутствует.

Если говорить о чисто фуллереновых объектах вообще, то, например, молекулярные кристаллы $\mathrm{C}_{60}$, характеризуясь шириной запрещенной зоны $E_{g}=1.5-2.3 \mathrm{eV}$ (по данным разных авторов [12]), считаются, как известно, полупроводниками $n$-типа с соответствующими температурными зависимостями $\rho(T)$. В то же время - это практически диэлектрики, у которых $\rho \approx 10^{6}-10^{7} \Omega \cdot \mathrm{cm}$. С ростом степени беспорядка $\rho$ возрастает. Во всех случаях в воздушной среде (т.е. при контакте с кислородом) $\rho$ увеличивается на 3-6 порядков и более, достигая $\sim 10^{14} \Omega \cdot \mathrm{cm}$. В полимерах, получаемых сжатием порошков $\mathrm{C}_{60}, \rho$ уменьшается, однако она вплоть до высоких давлений остается достаточно большой (соответственно электропроводность $\sigma$ остается довольно низкой), что создает экспериментальные трудности. Поэтому в таких образцах температурный ход $\rho$ или $\sigma$ измеряют, как правило, при $T>T_{R}$. Например, в работе [13] в образцах $\mathrm{C}_{60}$, полученных при давлениях $0.6-8 \mathrm{GPa}\left(T_{R}\right), \sigma$ практически не изменялась. При увеличении давления от 8 до $20 \mathrm{GPa}$ она увеличивалась на четыре порядка (от $2 \cdot 10^{-6}$ до $3 \cdot 10^{-2} \Omega^{-1} \cdot \mathrm{cm}^{-1}$ ). Температурные зависимости $\sigma$ в данной работе были измерены в диапазоне от $T_{R}$ до $400^{\circ} \mathrm{C}$. Для получения сведений об электропроводности фуллеренов при более низких температурах обычно используют пленки. Так что электропроводность наших образцов со 100\%-ным содержанием фуллеренов соответствует литературным данным, а образцы с самыми низкими относительными долями ТРГ имеют близкие величины $\sigma$ (рис. 1 ).

В целом наблюдаемое на рис. 1 поведение характерно для перколяционного механизма проводимости в неупорядоченном материале, состоящем из двух компонентов, один из которых - проводник, другой - изолятор. Теоретически механизм описывается степенным законом, представляющим собой зависимость проводимости материала от концентрации проводящего компонента в области перед переходом композита из проводящего состояния в изолирующее [14]. Иначе говоря,

$$
\sigma=\sigma_{0}\left(x-x_{c}\right)^{t},
$$

где $\sigma_{0}-$ константа, $x-$ концентрация проводящей фазы, $x_{c}-$ ее критическая концентрация или порог протекания, $t-$ критический индекс электропроводности, который обычно связан с размерностью объекта. Формула (1) справедлива при $x \geq x_{c}$, а при $x<x_{c}$ выполняется условие $\sigma=0$. Для трехмерных объектов, в том числе обладающих порами, теория предсказывает величину $t \approx 2$, что наблюдается в очень многих двухфазных материалах. Существенные отклонения от данного значения могут быть вызваны не только понижением размерности, но и другими причинами, например, контактными явлениями (в частности, если между проводящими элементами материала реализуются туннельные контакты вместо обычных омических [3]).

В нашем случае можно считать, что при $x \geq 5.9 \mathrm{wt} . \%$ (т.е. при относительной концентрации $\mathrm{C}_{60}$ от 0 до $94.1 \mathrm{wt. \% )}$ имеем металл, а при $x \leq 3.0 \mathrm{wt. \%}$ (т.е. при концентрации $\mathrm{C}_{60}$ от 97 до $\left.100 \mathrm{wt} . \%\right)$ - диэлектрик. Тогда в качестве порога протекания можно взять среднюю величину $x_{c}=4.45 \mathrm{wt} . \%$. 


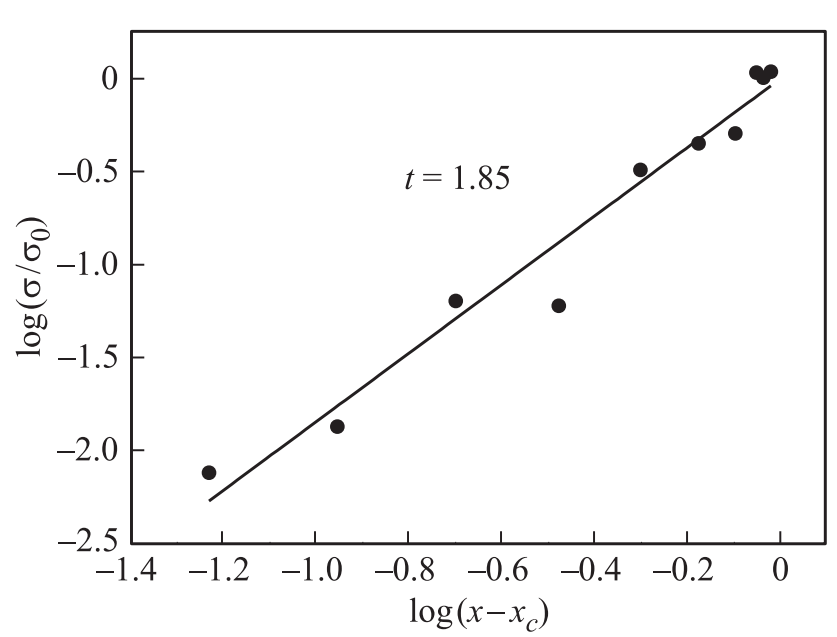

Рис. 3. Относительная удельная электропроводность композита $\sigma / \sigma_{0}$ как функция разности массовой доли ТРГ $(x)$ и величины порога протекания $\left(x_{c}=0.0445\right)$ в двойном логарифмическом масштабе; наклон прямой линии $t=1.85$.

Зависимость (1), построенная с учетом последнего обстоятельства в двойном логарифмическом масштабе (рис. 3), позволяет по наклону полученной прямой оценить параметр $t$, который оказывается равным $t=1.85$. Отметим, что, например, в композитах ТРГ-каменноугольный пек, полученных путем нагрева соответствующих дисперсных механических смесей до $200^{\circ} \mathrm{C}$, оценки дают значения $x_{c}=1.5 \%$ и $t=1.9$ [15], в композитах, где в полимерной матрице располагались сажевые частицы, $x_{c}=11 \%, t=2.24$ [16].

Если говорить о параметрах свободных носителей тока в металлической фазе нашего композита [9], то начальные значения их концентрации $n \approx 10^{20} \mathrm{~cm}^{-3}$ и подвижности $\mu \approx 30 \mathrm{~cm}^{2} \cdot \mathrm{V}^{-1} \cdot \mathrm{s}^{-1}$ (такие величины зафиксированы в чистом прессованном ТРГ и в образцах при соотношении $\left.\mathrm{C}_{60}: \mathrm{TP} \Gamma=1: 16\right)$. Непосредственно перед переходом металл-диэлектрик (т.е. при соотношении $\left.\mathrm{C}_{60}: \mathrm{TP} \Gamma=16: 1\right)$ каждый из указанных параметров снижается более чем на порядок, а именно до $n \approx 0.4 \cdot 10^{19} \mathrm{~cm}^{-3}$ и $\mu \approx 1 \mathrm{~cm}^{2} \cdot \mathrm{V}^{-1} \cdot \mathrm{s}^{-1}$, так что удельное сопротивление постепенно вырастает более чем на два порядка, как уже отмечалось. Можно предположить, что постепенный рост сопротивления образцов в рассматриваемой области происходит по двум причинам: вследствие убывания относительной доли проводящей фазы и роста количества разрывов в перколяционной сети. Убывание доли проводящей фазы ведет к снижению концентрации свободных носителей заряда, а одновременный рост количества разрывов (оборванных химических связей) в трехмерных проводящих сетях создает дополнительные ловушки, действующие как центры захвата подвижных носителей заряда и как центры рассеяния носителей, остающихся свободными. В итоге постепенно снижаются оба параметра, $n$ и $\mu$.

Дальнейшее падение доли проводящей фазы приводит к тому, что материал как бесконечный электропро- водящий кластер в какой-то момент перестает существовать, распадаясь на конечные электропроводящие фрагменты, электрически слабо связанные между собой. Постепенный рост сопротивления уже после перехода металл-диэлектрик и при дальнейшем приближении к чистому фуллереновому составу можно объяснить тем, что и в высокоомных образцах роль углеродной матрицы остается весьма заметной (несмотря на то что здесь величины $\rho$ определяются фуллеренами). В противном случае в образцах с наиболее высокими концентрациями $\mathrm{C}_{60}$ величина $\rho$, скорее всего, выходила бы на плато.

В связи с этим отметим еще одно обстоятельство. Неравномерность распределения проводящих и непроводящих фрагментов по объему материала может приводить к значительному разбросу величин электропроводности образцов как с одинаковым составом, так и при его вариациях, что наблюдается, например, в композитах типа углеродные нанотрубки-полимер [3]. В результате могут иметь место существенные отклонения от степенной зависимости типа (1). Что касается материала, представленного в настоящей работе, то косвенным подтверждением его удовлетворительной пространственной однородности (в указанном выше смысле) может служить достаточно низкий разброс параметров образцов (как правило, в пределах 10\%) и характер измеренных зависимостей. Например, в разных образцах чистого прессованного ТРГ $\rho=1.5-1.8 \mathrm{~m} \Omega \cdot \mathrm{cm}\left(T_{R}\right)$. Что касается характера зависимостей, то имеется в виду монотонность зависимости на рис. 1 до и после скачка $\rho$, одинаковая форма кривых на рис. 2 , не слишком сильный разброс экспериментальных точек на рис. 3. В этой связи зафиксировать рост сопротивления (причем достаточно плавный) на рис. 1 после перехода композита в высокоомное состояние можно, на наш взгляд, только при достаточной равномерности распределения в объеме материала проводящего компонента при самых низких его концентрациях. Отсутствие такой равномерности, вероятно, не позволило бы проявиться вкладу остатков углеродной матрицы в общую проводимость материала.

\section{5. Заключение}

В широком диапазоне относительных содержаний компонентов исследована электропроводность углеродуглеродного композиционного материала, в котором фуллерены $\mathrm{C}_{60}$ размещаются в проводящей матрице, полученной из терморасширенного графита. Образцы синтезированы путем термообработки исходных механических дисперсных смесей $\mathrm{C}_{60}+$ ТРГ в диффузионно-адсорбционном процессе и их последующего холодного прессования. В результате оба компонента оказываются распределенными (перемешанными друг с другом) по объему материала достаточно равномерно.

При увеличении относительного содержания $\mathrm{C}_{60}$ от 0 до 94.1 wt.\% удельное сопротивление композита постепенно вырастает на два порядка, при этом материал остается достаточно низкоомным и его можно отнести 
к металлическим системам со структурным беспорядком. При увеличении доли $\mathrm{C}_{60}$ от 94.1 до $97.0 \mathrm{wt} \%$ наблюдается резкий переход металл-диэлектрик ( $\rho$ увеличивается на 12 порядков). При дальнейшем росте концентрации $\mathrm{C}_{60}$ от 97.0 до $100 \mathrm{wt} . \% \rho$ постепенно увеличивается еще на порядок.

Делается вывод, что в низкоомных образцах электропроводность материала определяется главным образом углеродной матрицей на основе ТРГ, в высокоомных образцах - фуллеренами, при этом роль матрицы также остается заметной. В целом в композите наблюдается перколяционный механизм проводимости, характерный для материалов, состоящих из двух компонентов, один из которых - проводник, другой - изолятор.

Анализ, основанный на теории перколяции, показывает, что порог протекания как относительное содержание графитового компонента можно оценить значением $x_{c}=4.45 \mathrm{wt} . \%$, а оценка критического индекса электропроводности дает величину $t=1.85$, что характерно для двухкомпонентных трехмерных неупорядоченных сред, в том числе пористых.

\section{Список литературы}

[1] В.И. Березкин. Углерод: замкнутые наночастицы, макроструктуры, материалы. АРТЭГО, СПб (2013). 450 с.

[2] В.И. Березкин. Введение в физическую адсорбцию и технологию углеродных адсорбентов. Виктория плюс, СПб (2013). 409 c.

[3] А.В. Елецкий, А.А. Книжник, Б.В. Потапкин, Х.М. Кенни. УФН 185, 225 (2015).

[4] O. Gunnarson. Rev. Mod. Phys. 69, 575 (1997).

[5] V. Buntar, H.W. Weber. Supercond. Sci. Technol. 9, 599 (1996).

[6] В.И. Березкин. Письма в ЖЭТФ 83, 455 (2006).

[7] В.И. Березкин, В.В. Попов. ФТТ 49, 1719 (2007).

[8] В.И. Березкин, В.В. Попов, М.В. Томкович. ФТТ 59, 601 (2017).

[9] В.И. Березкин. ФТТ 59, 1432 (2017).

[10] В.В. Попов, Т.С. Орлова, J. Ramirez-Rico. ФТТ 51, 2118 (2009).

[11] В.Ф. Гантмахер. Электроны в неупорядоченных средах. Физматгиз, М. (2003). 176 c.

[12] Т.Л. Макарова. ФТП 35, 257 (2001).

[13] Y. Saito, H. Shinohara, M. Kato, H. Nagashima, M. Ohkohchi, Y. Ando. Chem. Phys. Lett. 189, 236 (1992).

[14] Б.И. Шкловский, А.А. Эфрос. Электронные свойства легированных полупроводников. Наука, М. (1979). 416 с.

[15] I.M. Afanasov, V.A. Morozov, A.V. Kepman, S.G. Ionov, A.N. Seleznev, G. Van Tendeloo, V.V. Avdeev. Carbon 47, 263 (2009).

[16] M. El Hasnaoui, M.P.F. Graça, M.E. Achour, L.C. Costa, A. Outzourhit, A. Oueriagli, A. E1 Harfi. J. Non-Cryst. Solids 356, 1536 (2010). 